\title{
A Comment on a Neuroimaging Study of Natural Language Quantifier Comprehension*广
}

\author{
Jakub Szymanik ${ }^{\ddagger}$ \\ Institute for Logic, Language, and Computation \\ University of Amsterdam \\ E-mail: szymanik@science.uva.nl \\ Plantage Muidergracht 24 \\ 1018 TV Amsterdam \\ The Netherlands
}

November 30, 2006

\section{Neuroimaging data}

Research presented in this journal by McMillan et al. (2005) is the first attempt to investigate the neural basis of natural language quantifiers (see also McMillan et al. (2006) for evidence on quantifier comprehension in patients with focal neurodegenerative disease and Clark and Grossman (2006) for more general discussion). It was devoted to study brain activity during comprehension of sentences with generalized quantifiers. Using BOLD fMRI the authors examined the pattern of neuroanatomical recruitment while subjects were judging the truth-value of statements containing natural language quantifiers. According to the authors their results verify a particular com-

\footnotetext{
${ }^{*}$ The author would like to express his appreciation to Theo Janssen for comments on the manuscript.

${ }^{\dagger}$ This research was supported by a Marie Curie Early Stage Research fellowship in the project GloRiClass (MEST-CT-2005-020841).

${ }^{\ddagger}$ The author is a recipient of the 2006 Foundation for Polish Science Grant for Young Scientists.
} 
putational model of natural language quantifier comprehension posited by several linguists and logicians (e. g. see van Benthem, 1986). We challenge this statement by evoking the computational difference between first-order quantifiers and divisibility quantifiers (e. g. see Mostowski, 1998). Moreover, we suggest other studies on quantifier comprehension, which can throw more light on the role of working memory in processing quantifiers.

\subsection{First-order and higher-order quantifiers}

The authors were considering the following two standard types of quantifiers: first-order and higher-order quantifiers. First-order quantifiers are those definable in first-order predicate calculus which is the logic containing only quantifiers $\exists$ and $\forall$ binding individual variables. In the research the following first-order quantifiers were used: "all", "some", and "at least 3". Higherorder quantifiers are those not definable in first-order logic. The subjects taking part in the experiment were presented with the following higher-order quantifiers: "less than half of", "an even number of", "an odd number of".

The expressibility of higher-order quantifiers is much greater than the expressibility of first-order quantifiers. For instance, we cannot speak about infinite sets in first-order logic, but this is possible using higher-order quantifiers. This difference in expressive power corresponds to the difference in the computational resources required to check truth-value of a sentence with those quantifiers.

In particular, to recognize first-order quantifiers we only need computability models which do not use any form of working memory. Intuitively, to check whether sentence (1) is true we do not have to remember anything.

(1) Every sentence in this paper is correct.

It suffices to read the sentences from this article one by one. If we find an incorrect one then we know that statement (1) is false. Otherwise, if we read the entire paper without finding any incorrect sentence, then statement (1) is true. We can proceed in a similar way for other first-order quantifiers. Formally, it was proved by Johan van Benthem (1986) that first-order quantifiers can be computed by such simple devices as finite automata. 
However, for recognizing some higher-order quantifiers, like "less than half" or "most", we need computability models making use of working memory. Intuitively, to check whether sentence (2) is true we must identify the number of correct sentences and hold it in working memory to compare with the number of incorrect sentences.

(2) Most of the sentences in this paper are correct.

Mathematically speaking, such algorithm can be realized by a push-down automaton.

From this perspective the authors hypothesize that all quantifiers recruit the right inferior parietal cortex which is associated with numerosity. Taking distinction on complexity of first-order and higher-order quantifiers for granted they also predict that only higher-order quantifiers recruit the prefrontal cortex which is associated with executive resources, like working memory. In other words, they believe that the computational complexity differences between first-order and higher-order quantifiers are also reflected in brain anatomy during processing quantifier sentences (McMillan et al., 2005, p. 1730). This hypothesis was confirmed.

In the next section I will discuss these results and show that the distinction between first-order and higher-order quantifiers is not sufficient if one wants to investigate the role of working memory in quantifier comprehension.

\section{Discussion}

In my view the authors interpretation of their results is not convincing. Also, their experimental design may not provide the best means of differentiating between the neural bases of the various kinds of quantifiers. The main point of criticism is that the distinction between first-order and higher-order quantifiers does not coincide with the computational resources required to compute the meaning of quantifiers. There is a proper subclass of higherorder quantifiers, namely divisibility quantifiers, which corresponds - with respect to working memory - to exactly the same computational model as first-order quantifiers. Let us have a closer look into the paper of McMillan et al. (2005). 


\subsection{Quantifiers and working memory}

The authors suggest that their study honours a distinction in complexity between classes of first-order and higher-order quantifiers. They also claim that:

higher-order quantifiers can only be simulated by a more complex computing device - a push-down automaton - which is equipped with a simple working memory device. (McMillan et al., 2005, p. 1730)

Unfortunately, this is not completely true. Most of the quantifiers qualified in the research as higher-order quantifiers can be recognized by finite automata. Both "an even number" and "an odd number" are quantifiers recognizable by two-state finite automata with transition from the first state to the second and vice versa. In the case of the automaton corresponding to "even" the initial state is also the accepting state. In the automaton for "odd" the other state is the accepting one. Intuitively, to check whether sentence (3) is true you do not need to count the number of incorrect sentences and then compare it with that of the set of even integers.

(3) An even number of the sentences in this paper is incorrect.

You need only remember parity. For example when you find an incorrect sentence you write "1" at the blackboard, if you find another one you erase "1" and put "0" again, then if you see another incorrect sentence you put "1" in place of "0", and so on. At every moment you have only one digit at the blackboard no matter how long is the paper.

In what follows we give a short description of relevant mathematical results. Quantifiers definable in first-order logic, FO, can be recognized by acyclic finite automata, which are a proper subclass of the class of all finite automata (van Benthem, 1986). Less known result due to Marcin Mostowski (1998) says that exactly the quantifiers definable in divisibility logic, $F O\left(D_{n}\right)$, (i.e. first-order logic enriched by all quantifiers "divisible by $n$ ", for $n \geq 2$ ) are recognized by finite automata (FA) . For instance, quantifier $D_{2}$ can be use to express the natural language quantifier "an even number of". 
Quantifiers of type (1) not definable in $F O\left(D_{n}\right)$ but expressible in the arithmetic of addition, Pr (Presburger Arithmetic), are recognized by pushdown automata, PDA, (van Benthem, 1986). Push-down automata are computability models making essential use of working memory in the form of so-called stack.

Obviously, semantics of many natural language quantifier expressions can not be modeled by such simple devices as PDA. Just think about sentence (4) which meaning cannot be computed by any PDA what easily follows from Pumping Lemma for context-free languages.

(4) An equal number of logicians, philosophers, and linguists climbed Elbrus.

There are even much more complex expressions in natural language. For example, consider quantifiers definable in the existential fragment of secondorder logic, $\Sigma_{1}^{1}$, like those in sentences (5) and (6).

(5) Some relative of each villager and some relative of each townsman hate each other.

(6) Most of villagers and most of townsmen hate each other.

It was shown by Mostowski and Wojtyniak (2004) and Sevenster (2006) that both sentences are NP-complete, i. e. computable by nondeterministic Turing Machine, TM, in polynomial time. What is important is summed up in table 1 (for a general discusson on semantical bounds for natural language expressibility see also Mostowski and Szymanik (2005)).

\begin{tabular}{|c|c|c|}
\hline definability & example & recognized by \\
\hline FO & "all cars", "some students", "at least 3 balls" & acyclic FA \\
\hline$F O\left(D_{n}\right)$ & "an even number of balls" & FA \\
\hline $\operatorname{Pr}$ & "most lawyers", "less than half of the students" & PDA \\
\hline$\Sigma_{1}^{1}$ & "most of ... and most of ..." & TM \\
\hline
\end{tabular}

Table 1: Quantifiers and complexity of corresponding algorithms.

You could see that our main point of criticism is justified. It is not the case that first-order and higher-order quantifiers differ with respect to 
working memory requirement. Therefore, the explanation of brain activation patterns proposed by the authors is based on the wrong assumption. A simple automata-theoretic perspective is not enough to describe the processing of natural language quantifiers. Some additional arguments need to be found for interpreting the results. In what follows I will propose a few ways of empirical exploration into the subject.

\section{Improving experiment}

\subsection{First-order and divisibility quantifiers}

We should compare brain activation with respect to the three classes of quantifiers: recognizable by acyclic FA (first-order), FA (divisibility), and PDA. I do not know whether the authors compare these classes. If they did, then it would be important to analyze it. The authors did not report any data on these differences.

Particularly, we predict differences between first-order and divisibility quantifiers. Comprehension of divisibility quantifiers - but not first-order quantifiers - should depend on the executive resources (dorsolateral prefrontal cortex). It would correspond then to the difference between acyclic finite automata and finite automata.

We expect that only quantifiers not definable in divisibility logic will activate working memory (inferior frontal cortex).

\subsection{Aristotelean and cardinal quantifiers}

It would be also interesting to compare Aristotelean quantifiers, like "all", "every", "some", "no", "not all", with cardinal quantifiers, e. g. "at least 3", "at most 7", "between 8 and 11". They are all definable in first-order logic, but elementary representation of cardinal quantifiers can be ill-suited for psychological purposes. Consider for example how "at least 3 balls" is translated into first-order logic:

$$
\exists x \exists y \exists z(x \neq y \wedge y \neq z \wedge \operatorname{ball}(x) \wedge \operatorname{ball}(y) \wedge \operatorname{ball}(z)) .
$$

Since we cannot talk about sets in elementary logic, then - as you can deduce from above example - the complexity of first-order translation of 
cardinality quantifiers is proportional to the rank of the cardinal that needs to be represented.

In the reported study, only one cardinal quantifier of relatively small rank was taken into consideration, namely "at least 3". It might be the case that with respect to mental processing complexity of cardinal quantifiers is more similar to that of higher-order quantifiers than to Aristotelean. However, to observe this we should use cardinal quantifiers of higher rank, for instance "at least 7". Obviously, this issue is strongly connected with the phenomena of subitizing as opposed to counting.

\subsection{Quantifiers and ordering}

Finally, there are many possible ways of verifying the role of working memory in natural language quantifier processing. One way is as follows. In the reported research, subjects were presented sentences with visual arrays and have to decide whether a sentence is true at it. Elements at arrays were randomly generated. However, ordering of elements can be treated as an additional independent variable to investigate the role of working memory. For example, consider the following sentence:

(7) Most As are B.

Although checking the truth-value of sentence (7) over an arbitrary universe needs use of a kind of working memory, if the elements of a universe are ordered in pairs $(a, b)$ such that $a \in A, b \in B$, then we can easily check it without using working memory. It suffices to go through the universe and check whether there exists an element $a$ not paired with any $b$. This can be done by a finite automaton. It would be interesting to carefully compare the pattern of neuroanatomic recruitment while subjects are judging the truthvalue of statements, like sentence (7), over ordered and arbitrary universes. We predict that when dealing with ordered universe the working memory will not be activate, but it will if the elements are placed in arbitrary way. 


\section{Conclusion}

We discussed the research by McMillan et al. (2005) on neural basis of quantifiers comprehension. We have shown that logical distinction on firstorder and higher-order quantifiers used by the authors is not sufficient for investigating the role of working memory in quantifier processing. The main point of our criticism is that this distinction does not coincide with the need of using working memory for computing denotation of quantifiers. We finished the paper by giving few suggestions on improved experiment.

\section{References}

Clark, R. and Grossman, M. (2006). Number sense and quantifier interpretation. Submitted.

McMillan, C., Clark, R., Moore, P., Devita, C., and Grossman, M. (2005). Neural basis for generalized quantifiers comprehension. Neuropsychologia, 43:1729-1737.

McMillan, C., Clark, R., Moore, P., and Grossman, M. (2006). Quantifiers comprehension in corticobasal degeneration. Brain and Cognition, 65:250260.

Mostowski, M. (1998). Computational semantics for monadic quantifiers. Journal of applied Non-Classical Logics, 8:107-121.

Mostowski, M. and Szymanik, J. (2005). Semantical bounds for everyday language. Semiotica, to appear.

Mostowski, M. and Wojtyniak, D. (2004). Computational complexity of the semantics of some natural language constructions. Annals of Pure and Applied Logic, 127:219-227.

Sevenster, M. (2006). Branching Imperfect Information. Logic, Language, and Computation. PhD thesis, Institute for Logic, Language, and Computation.

van Benthem, J. (1986). Essays in logical semantics. Reidel. 